\title{
Journal of Physiotherapy \& Physical Rehabilitation
}

\section{Prevalence of Scapular Dyskinesis in Patients with Distal Radius Fracture with or without Shoulder Pain}

Héctor Gutiérrez-Espinoza ${ }^{1,2^{*}}$, Cristian Olguín-Huerta ${ }^{1}$, Jonathan Zavala-González ${ }^{1,2}$, David Rubio-Oyarzún ${ }^{1}$, Felipe Araya-Quintanilla ${ }^{1}$, Mario RiosRiquelme $^{1,3}$ and Nicolle Valenzuela-Briones ${ }^{1}$

${ }^{1}$ Physical Therapy School, University of the Americas, Santiago, Chile

${ }^{2}$ Department of Physical Therapy, Clinical Hospital San Borja Arriaran, Santiago, Chile

${ }^{3}$ Bachelor of Sciences in Physical Activity, University of the Santiago, Santiago, Chile

"Corresponding author: Héctor Gutiérrez-Espinoza. Physical Therapy School, University of the Americas, Echaurren Street 140, 3rd floor, Santiago, Chile 8320000, Tel: +56-992999298, E-mail: kinehector@gmail.com

Received date: April 24, 2017; Accepted date: May 02, 2017; Published date: May 08, 2017

Copyright: () 2017 Guetierrez-Esponza H, et al. This is an open-access article distributed under the terms of the Creative Commons Attribution License, which permits unrestricted use, distribution, and reproduction in any medium, provided the original author and source are credited.

\begin{abstract}
Objective: To describe prevalence of scapular dyskinesis (SD) in adult patients with distal radius fractures (DRF).

Material and methods: The current cross-sectional study was conducted at the San Borja Arriaran Clinical Hospital. 180 patients with a type an extra-articular DRF, according to the AO classification, were recruited. The presence of SD was evaluated with the yes/no dynamic test based on visual criteria, and the clinical diagnosis of shoulder pain was performed by orthopedic surgeon.

Results: The prevalence of SD is shown in $80 \%$ of all patients with DRF, this value increases to $90.9 \%$ in group of patients with associated shoulder pain. The odds ratio was 10 with a $95 \% \mathrm{Cl}: 4.1-24.8(p=0.0000)$.

Conclusions: The prevalence of SD is shown in $80 \%$ of all patients with DRF; this value increases to $90.9 \%$ in the group of patients that present DRF with associated SP. The ratio for estimating prevalence is 10 times higher in patients with DRF with shoulder pain compared with the asymptomatics shoulders. There is a statistically significant association between the presence of SD and shoulder pain in patients with DRF.
\end{abstract}

Keywords: Distal radius fracture; Scapular dyskinesis; Shoulder pain; Cross-sectional study; Odds ratio

\section{Introduction}

Distal radius fracture (DRF) is the most frequent fracture in adults, representing about $20 \%$ of total fractures treated in emergency services [1], epidemiologic studies have reported high incidence in white populations, especially in elderly patients [2,3]. DRFs in patients have typically been treated conservatively with closed reduction and plaster cast immobilization [4]. The damage caused by a fracture and the associated immobilization, leaves some lasting effects, such as pain, edema, reduced range of motion, decreased strength, deformation, and impairment of injured limb function [5].

The reported complication rates in relation with DRF are highly variable [6]. McKay et al. reviewed the overall incidence of complications after DRF in the literature, loss of motion, delay in consolidation and/or vicious consolidation, Dupuytren's contracture, complex regional pain syndrome type 1, and secondary arthritis/ arthrosis present the highest levels of incidence [7].

Most of the complications mentioned above are located in the surrounding areas of the original injury; however, compensatory motions at proximal segments following a distal injury have been reported several studies [8-12]. It should be noted that the scapula is one of the most affected anatomical structures by these adaptive changes, because the scapula acts as a linkage between the trunk and the Upper Extremities (UE), it is therefore more vulnerable to the deleterious effects of distal injuries [13].

The role of the scapula is essential for an optimum performance of the UE $[14,15]$, optimal positioning of the scapula is essential for enhancing the quality of arm movement when fine motor control is required to achieve goal-directed activities $[14,16]$. Scapular motion provides optimal muscle length-tension ratios for accurate movement patterns and promotes muscular energy conservation during arm motion [14]. In particular, the scapulothoracic joint creates a functional bridge between the upper extremity and the trunk segment, where $51 \%$ of the kinetic energy is sequentially transferred to distal segments [14].

Alterations in the normal kinematics of the scapula are called Scapular Dyskinesis (SD) $[17,18]$. It is a general term that reflects the loss of normal control of scapular motion [17]. There is substantial evidence of scapular kinematics in people with shoulder pain, across a variety of shoulder pathologies [17-19], such as subacromial impingement syndrome [20,21], glenohumeral instability [22], adhesive capsulitis [20], tendinopathy and/or rotator cuff tears [20], and clavicle fractures [23].

Despite the SD may be found in association with many types of shoulder pathologies, the exact relationship between dyskinesis and clinical pathology is not clear $[17,18]$. SD is a non-specific response to a painful condition in the shoulder rather than a specific response to certain glenohumeral pathology; therefor it is more appropriate to consider it a potential impairment to shoulder function [17]. 
Citation: Gutiérrez-Espinoza H, Olguín-Huerta C, Zavala-González J, Rubio-Oyarzún D, Araya-Quintanilla F, et al. (2017) Prevalence of Scapular Dyskinesis in Patients with Distal Radius Fracture with or without Shoulder Pain. Physiother Rehabil 2: 140. doi: $10.4172 / 2573-0312.1000140$

Page 2 of 5

Recently the study published by Ayhan et al. [13] showed that patients with DRF present a significant increase in the upward and internal rotation of the scapula during arm elevation in the frontal plane compared to asymptomatic subjects; and present an increment in the upward rotation during scapular plane elevation and there was an increase in the anterior tilting elevation in the sagittal plane [13].

These changes in the normal kinematics of the scapula lead to some consequences, like pain and limited range of motion in the shoulder in patients with DRF. There are, however, no published studies that have reported rates of incidence and/or prevalence of Shoulder Pain (SP) in patients with distal radius fracture. SP is a common complaint in primary health care resulting in significant pain, disability, loss of productivity and health care costs [24]. The lifetime prevalence of SP in the general population is reported between $6.7 \%$ to $66.7 \%$ [25].

The main objective of this current investigation is to describe the prevalence of SD en patients with DRF with or without shoulder pain and the secondary objective is to determine if there is correlation between the presence of SD and the presence of associated Shoulder Pain.

\section{Material and Method}

The current descriptive cross-sectional study was conducted at the San Borja Arriaran Clinical Hospital; the Ethics Committee of the Servicio de Salud Metropolitano Central in Chile approved the study protocol. Between 2012 and 2016, the study was performed using a non-probabilistic sampling method, 180 adult patients with an extraarticular DRF were recruited consecutively.

The AO classification was used to evaluate the fracture type [26]. All patients were treated with closed reduction and plaster cast immobilization for 6 weeks, after cast removal the presence of SP was evaluated. The clinical diagnosis was performed by an orthopedic surgeon, the positive findings were painful arc of movement during flexion or abduction; positive Neer or Hawkins-Kennedy impingement signs; pain or resisted lateral rotation, abduction or empty cam test [27].

Subsequent to cast removal all patients were prescribed acetaminophen $500 \mathrm{mg}$ every 8 hours for 10 days and were referred for physical therapy. In the first evaluation, the clinical assessment included an evaluation to identify the presence of SD through the yes/no dynamic test based on visual criteria proposed by Uhl et al. [28], there was an informed consent accepted by the subject.

\section{Inclusion and exclusion criteria}

The following patient inclusion criteria were applied:

Adult patients were forwarded from the Department of Orthopaedic Surgery at the San Borja Arriaran Clinical Hospital. These patients were diagnosed with a type an extra-articular DRF, according to the AO classification; and treated conservatively with closed reduction and plaster cast immobilization.

\section{Informed consent accepted and signed by the subject}

The following patient exclusion criteria were applied:

Patients that showed immediate complications after the removal of the immobilization (Complex regional pain syndrome type 1, Carpal tunnel syndrome, etc.).
Patients diagnosed with shoulder pathology in the course of the 1 year period before presenting DRF (sub-acromial impingement syndrome, partial or complete rotator cuff tear, glenohumeral instability, adhesive capsulitis, etc.).

\section{Evaluation}

All patients were evaluated before treatment; the evaluation was performed by a physical therapy, not associated with the research team, who had a Master's degree in Orthopedic Manual Therapy and more than ten years of clinical experience.

The current recommendation for the clinical evaluation of SD, based on a consensus meeting, is the use of dynamic scapular dyskinesis test $[17,18]$. The presence of SD was evaluated through the yes/no dynamic test based on visual criteria proposed by Uhl et al. [28], they simplified the original method published by Kibler [29], characterizing any abnormal movement of the scapula with a Yes (Kibler Type 1,2, and 3 classification system) and the absence of abnormality with a No (Kibler Type 4 classification system).

Specifically, clinical observation of the medial and inferior scapular borders for winging or medial border prominence, lack a smooth coordinated movement as exemplified by early scapular elevation or shrugging during ascending arm forward flexion, and rapid downward rotation during arm lowering from full flexion [17].

The motion is then characterized as dyskinesis as a "Yes" (presence of winging and/or dysrhythmia) or "No" (no presence) [28].

\section{Statistical analysis}

All collected data was entered into Excel for tabulation, and the statistical analysis was performed using Stata IC 11.0. A $2 \times 2$ contingency table was used to provide bivariate analysis of studied dichotomous variables, the independent variable was placed in the rows (presence or absence of SD) and the dependent variable is placed in the columns (presence or absence of SP).

Prevalence was used as a measure of frequency in all DRF patients and in the group with associated SP. "Odds ratio" (OR) was used as a measure of association. Pearson's chi-squared test was used to determine whether there is correlation between the presence of SD and the presence of SP associated to patients with DRF.

\section{Results}

The results of the characteristics of the studied group are shown in Table 1. The average patient age was 57.1 years $(23.2), 80.6 \%$ of the samples were women. All patients were treated with closed reduction and plaster cast following 6.4 weeks of immobilization period and $70 \%$ were affected on the dominant side.

In regards to the extra-articular sub-type of fracture, according to AO classification, $71.7 \%$ presented an A3 DRF ( $n=129), 23.3 \%$ presented an A2 DRF $(n=42)$ and the other $5 \%$ an A1 ( $n=9)$.

In regards to the total sample of 180 patients with DRF, there is a prevalence of $80 \%$ of patients with SD. Subsequent to cast removal, $73.3 \%$ of the total (132 patients) were clinical diagnosed with SP. 
Citation: Gutiérrez-Espinoza H, Olguín-Huerta C, Zavala-González J, Rubio-Oyarzún D, Araya-Quintanilla F, et al. (2017) Prevalence of Scapular Dyskinesis in Patients with Distal Radius Fracture with or without Shoulder Pain. Physiother Rehabil 2: 140. doi: 10.4172/2573-0312.1000140

Page 3 of 5

\begin{tabular}{|l|l|}
\hline Parameters & Patients with DRF (n=180) \\
\hline Gender female (number \%) & $145 / 180(80.6 \%)$ \\
\hline Age (years), mean (SD) & $57.1(23.2)$ \\
\hline Immobilization time (weeks), mean (DE) & $6.4(0.8)$ \\
\hline Fractured dominant hand (number \%) & $126 / 180(70 \%)$ \\
\hline DRF A1 according to AO (number \%) & $9 / 180(5 \%)$ \\
\hline DRF A2 according to AO (number \%) & $42 / 180(23.3 \%)$ \\
\hline DRF A3 according to AO (number \%) & $129 / 180(71.7 \%)$ \\
\hline Shoulder pain (number \%) & $132 / 180(73.3 \%)$ \\
\hline Scapular dyskinesis test (+) (number \%) & $144 / 180(80 \%)$ \\
\hline DRF: Distal Radius Fracture; SD: Standar Deviation & \\
\hline
\end{tabular}

Table 1: Baseline characteristics of patients with extra-articular DRF.

Considering only subjects that were previously diagnosed with SP, presence of SD rises to $90.9 \%$. Examining the results of the contingency table (Table 2), we were able to determine the OR as a measure of association between the presence of SD and the clinical diagnosis of SP with a total sample of 180 patients. $132(73.3 \%)$ presented associated SP and 48 (26.7\%) were asymptomatic, when looking specifically at patients with a positive Uhl dynamic test for SD in each of the two groups (both positive and negative for SP), the OR is 10 , with a $95 \%$ CI $4.1-24.8$ ( $\mathrm{p}=0.0000)$. The results of the chi-squared test show that there is a statistically significant correlation $(\mathrm{p}=0.000)$ between the presence of SD and SP, according to the Cramer's V results $(\mathrm{V}=0.69)$, the magnitude of the association between the variables is high.

\begin{tabular}{|l|l|l|l|l|}
\hline \multirow{2}{*}{\multicolumn{2}{|l|}{}} & \multicolumn{2}{l|}{ Shoulder Pain } & \multirow{2}{*}{ Total } \\
\cline { 3 - 5 } & & Yes & No & \\
\hline $\begin{array}{l}\text { Scapular Dyskinesis } \\
\text { Test }\end{array}$ & Yes & 120 & 24 & 144 \\
\cline { 2 - 5 } & No & 12 & 24 & 36 \\
\hline Total & & 132 & 48 & 180 \\
\hline
\end{tabular}

Table 2: Contingency table for scapular dyskinesis/shoulder pain.

\section{Discussion}

In the current cross-sectional study, researchers were able to describe the prevalence of SD, which was evaluated through the yes/no dynamic test proposed by Uhl et al. [28] A group of adult patients with extra-articular DRF, according to AO, treated conservatively with closed reduction and plaster cast immobilization were evaluated. This is exploratory research, there are no similar studies published, and the review of the literature revealed that there was an article by Ayhan et al. [13] published, which evaluated kinematics of the scapula in 16 patients with DRF with an electromagnetic system. To compare data with the asymptomatic control group, they showed changes in the three planes of scapular kinematic movements.
Knowledge regarding the roles of scapula in shoulder function has been gradually accumulating [17], scapular position and motion are closely integrated with arm motion to accomplish most shoulder functions [30]. These roles included providing synchronous scapular rotation during humeral motion, serving as a stable base for rotator cuff activation and functioning as a link in the kinetic chain [17,29,31]. Scapular dyskinesis ("dys"-alteration of, "kinesis"-movement) is a collective term that refers to movement of the scapula that is dysfunctional $[17,18,30]$. SD has been identified by a group of experts as: (1) abnormal static scapular position and/or dynamic scapular motion characterized by medial border prominence; or (2) inferior angle prominence and/or early elevation or shrugging on arm elevation; and/or (3) rapid downward rotation during arm lowering $[18,30]$. However, static position and dynamic motion are two separate entities, so when describing the static appearance of the scapula and if asymmetry is observed, it should be referred to as 'altered scapular resting position' rather than SD [30]. Clinical evaluation of scapular dysfunction in patients with shoulder pain should include the visual observation to determine the presence or absence of SD [17]. Visual dynamic assessment schemes of classifying the presence of SD during the shoulder motion have been developed in an attempt to resolve the issues with linear or static measures $[28,29]$. These methods are considered more functional and more inclusive with the ability to judge scapular movement in three-dimensional patterns [17,18,31].

Although it is clear that this investigation cannot establish direct causality between the variables of the study, the results show a high prevalence of SD in patients with DRF and associated SP, compared to asymptomatic shoulder in patients with DRF, the clinical method used to detect presence or absence of SD was the yes/no dynamic test proposed by Uhl et al. [28]. It showed a $79 \%$ inter-rated correlation using this system $(\mathrm{k}=0.41)$ and it has sensitivity values between 74 and $78 \%$, whereas specificity values ranged between 31 and $38 \%$ when using asymmetry found with three-dimensional testing as a gold standard [28]. It is a simple evaluation method, it is inclusive (does not force the evaluator to choose one type of alteration) and recommended for clinical use $[17,18,31]$, it is a method that improves the psychometric properties in symptomatic patients despite the regular specificity in their values [31]. As an evaluation, patients were asked to perform active movements during arm elevation in a frontal, scapular, 
Citation: Gutiérrez-Espinoza H, Olguín-Huerta C, Zavala-González J, Rubio-Oyarzún D, Araya-Quintanilla F, et al. (2017) Prevalence of Scapular Dyskinesis in Patients with Distal Radius Fracture with or without Shoulder Pain. Physiother Rehabil 2: 140. doi: $10.4172 / 2573-0312.1000140$

Page 4 of 5

and sagittal plane. Any sign of winging and/or dysrhythmia in the ipsilateral side of the DRF was categorized with a 'yes'. Alterations in the kinematics of the scapula must be evaluated in different planes $[17,18,28]$, because prevalence of asymmetric evaluation in only one plane has not shown significant differences between symptomatic and asymptomatic patients [28]. Uhl et al. publication and in our study, the highest percentage of alterations in kinematics (presence of winging and/or dysrhythmia) was seen in the anterior active flexion movement of the arm in the frontal plane. 117 out of 144 patients with SD $(81.3 \%)$ presented alteration while performing this action.

Regarding the results of the correlation, there is a high association and statistical significance between the presence of SD that was evaluated though a yes/no dynamic test and the patients that present SP ipsilateral to the DRF, only 22 out of 132 patients with the clinical diagnosis of SP post DRF were symptomatic after a physical therapy treatment. Clinical assessment included a radiological study and ultrasound for the diagnosis of damage to the soft tissues of the shoulder. 18 of the participants were diagnosed with supraspinatus tendinosis and subacromial bursitis, 2 with partial a tear of the supraspinatus and signs of infraspinatus tendinosis, and 2 with total tear of the supraspinatus and tenosynovitis of the long portion of the bicep. All participants were treated conservatively and had a positive response to medical treatment.

Our study presents some limitations. The cross-sectional studies are descriptive; they compile data in a determined moment and do not permit the establishment of a temporal sequence of events between the variables of the study (cause-effect), this is a non-probabilistic sampling method because this is exploratory research, the data related to the prevalence of SD in the totality of patients with DRF is unknown, and the diagnosis of SP was made with the information from clinical examination. After the removal of immobilization, patients were not given imaging exams. The evaluation tool used to detect the presence of SD is one of the most recommended for clinical usage, it is not however the "gold standard" for diagnosing this condition, but has shown good inter-rated reliability and high percentages of sensitivity, and has regular specificity in its values. It is however important to mention that there is inherent difficulty in the diagnosis of SP as a product of DRF. Although we did gather our information from clinical records, we carried out meticulous anamnesis, and this can always be a source of bias, as such the results of this study should be interpreted with caution.

\section{Conclusions}

The prevalence of SD is shown in $80 \%$ of all patients with DRF, this value increases to $90.9 \%$ in the group of patients that present DRF with a clinical diagnosis of associated SP. The odds of having and not having SD are 10 times higher in patients with DRF with SP compared to patients with an asymptomatic shoulder. There is a statistically significant association between the presence of SD and SP in patients with DRF.

\section{References}

1. Bengnér U, Johnell O (1985) Increasing incidence of forearm fractures. A comparison of epidemiologic patterns years apart. Acta Orthop Scand 56: 158-160.

2. Sahlin Y (1990) Occurrence of fractures in a defined population: A 1-year study. Injury 21: 158-160.
3. Singer BR, McLauchlan GJ, Robinson CM, Christie J (1998) Epidemiology of fractures in 15,000 adults: The influence of age and gender. J Bone Joint Surg Br 80: 243-248.

4. Chung KC, Shauver MJ, Birkmeyer JD (2009) Trends in the Unites States in the treatment of distal radius fractures in the elderly. J Bone Joint Surg Am 91: 1868-1873.

5. Gutiérrez H, Herrera U, Aguilera R, Gutiérrez R (2011) Physiotherapy in distal radius fractures: Systemic revision. Rev Iberoam Fisioter Kinesiol 14: 25-37.

6. Turner RG, Faber KJ, Athwal GS (2010) Complications of distal radius fractures. Hand Clin 26: 85-96.

7. McKay SD, MacDermid JC, Roth JH, Richards RS (2001) Assessment of complications of distal radius fractures and development of a complication checklist. J Hand Surg Am 26: 916-922.

8. Ayhan C, Unal E, Yakut Y (2014) Core stabilisation reduces compensatory movement patterns in patients with injury to the arm: a randomized controlled trial. Clin Rehabil 28: 36-47.

9. Bulthaup S, Cipriani DJ, Thomas JJ (1999) An electromyography study of wrist extension orthoses and upper-extremity function. Am J Occup Ther 53: 434-440.

10. Carey SL, Highsmith MJ, Maitland ME, Dubey RV (2008) Compensatory movements of transradial prosthesis users during common tasks. Clin Biomech (Bristol, Avon) 23: 1128-1135.

11. Yoo IG, Jung MY, Jeon HS, Lee J (2010) Effects of wrist-extension orthosis on shoulder and scapular muscle activities during simulated assembly tasks. Ind Health 48: 108-114.

12. King S, Thomas JJ, Rice MS (2003) The immediate and short-term effects of a wrist extension orthoses on upper-extremity kinematics and range of shoulder motion. Am J Occup Ther 57: 517-524.

13. Ayhan C, Turgut E, Baltaci G (2015) Distal radius fractures result in alterations in Scapular Kinematics: a three-dimensional motion analysis. Clin Biomech (Bristol, Avon) 30: 296-301.

14. Kibler WB (1998) The role of the scapula in athletic shoulder function. Am J Sport Med 26: 325-337.

15. Voight ML, Thomson BC (2000) The role of the scapula in the rehabilitation of shoulder injuries. J Athl Train 35: 364-372.

16. Paine R, Voight ML (2013) The role of the scapula. Int J Sports Phys Ther 8: 617-629.

17. Kibler WB, Ludewig PM, McClure PW, Michener LA, Bak K, et al. (2013) Clinical implications of scapular dyskinesis in shoulder injury: The 2013 consensus statement from the "Scapular Summit". Br J Sport Med 47: 877-885.

18. Kibler BW, Ludewig PM, McClure P, Uhl TL, Sciascia A (2009) Scapular summit 2009: Introduction. J Orthop Sports Phys 39: A1-A13.

19. Kibler WB, Sciascia A, Wilkes $T$ (2012) Scapular dyskinesis and its relation to shoulder injury. J Am Acad Orthop Surg 20: 364-372.

20. Ludewig PM, Reynolds JF (2009) The association of scapular kinematics and glenohumeral joint pathologies. J Orthop Sports Phys 39: 90-104.

21. Timmons MK, Thigpen CA, Seitz AL, Karduna AR, Arnold BL, et al. (2012) Scapular kinematics and subacromial-impingement syndrome: A meta-analysis. J Sport Rehabil 21: 354-370.

22. Kibler WB, Sciascia A (2016) The role of the scapula in preventing and treating shoulder instability. Knee Surg Sports Traumatol Arthrosc 24: 390-397.

23. Shields E, Behrend C, Beiswenger T, Strong B, English C, et al. (2015) Scapular dyskinesis following displaced fractures of the middle clavicle. J Shoulder Elbow Surg 24: 331-336.

24. Virta L, Joranger P, Brox JL, Eriksson R (2012) Costs of shoulder pain and resource use in primary health care: A cost-of-illness study in Sweden. BMC Musculoskelet Disord 10: 13-17.

25. Luime JJ, Koes BW, Hendriksen IJM, Burdof A, Verhagen AP, et al. (2004) Prevalence and incidence of shoulder pain in the general population: A systematic review. Scand J Rheumatol 33: 73-81. 
Citation: Gutiérrez-Espinoza H, Olguín-Huerta C, Zavala-González J, Rubio-Oyarzún D, Araya-Quintanilla F, et al. (2017) Prevalence of Scapular Dyskinesis in Patients with Distal Radius Fracture with or without Shoulder Pain. Physiother Rehabil 2: 140. doi: $10.4172 / 2573-0312.1000140$

Page 5 of 5

26. Kreder HJ, Hanel DP, McKee M, Jupiter J, McGillivary G, et al. (1996) Consistency of $\mathrm{AO}$ fractures classification for the distal radius. J Bone Joint Surg Br 78: 726-731.

27. Hegedus EJ, Goode AP, Cook CE, Michener L, Myer CA, et al. (2012) Which physical examination tests provide clinicians with the most value when examining the shoulder? Update of a systematic review with metaanalysis of individuals tests. Br J Sports Med 46: 964-978.

28. Uhl TL, Kibler BW, Gecewich B, Tripp BL (2009) Evaluation of clinical assessment methods for scapular dyskinesis. Arthroscopy 25: 1240-1248.
29. Kibler WB, Uhl TL, Maddux JWQ, Brooks PV, Zeller B, et al. (2002) Qualitative clinical evaluation of scapular dysfunction: A reliability study. J Shoulder Elbow Surg 11: 550-556.

30. Kibler WB, Sciascia A (2010) Current concepts: Scapular dyskinesis. Br J Sports Med 44: 300-305.

31. Gutiérrez H, Cereceda C, Olguín C, Jordán R, Gana G (2015) Validity and reliability of the evaluation of scapular dyssinesis through visual criteria: a review of the literature. Rev Chil Ort y Traum 56: 33-45. 